\title{
The European Consensus on grading of bone marrow fibrosis allows a better prognostication of patients with primary myelofibrosis
}

Umberto Gianelli ${ }^{1,6}$, Claudia Vener ${ }^{2,6}$, Anna Bossi ${ }^{3}$, Ivan Cortinovis ${ }^{3}$, Alessandra Iurlo ${ }^{4}$, Nicola S Fracchiolla ${ }^{4}$, Federica Savi ${ }^{1}$, Alessia Moro ${ }^{5}$, Federica Grifoni ${ }^{4}$, Chiara De Philippis ${ }^{4}$, Tommaso Radice ${ }^{4}$, Silvano Bosari ${ }^{1}$, Giorgio Lambertenghi Deliliers ${ }^{4}$ and Agostino Cortelezzi ${ }^{4}$

${ }^{1}$ U.O.C di Anatomia Patologica, Università degli Studi di Milano, Dipartimento di Medicina, Chirurgia e Odontoiatria, Fondazione IRCCS Ca’ Granda-Ospedale Maggiore Policlinico, Milan, Italy; ${ }^{2}$ Centro Trasfusionale e Immunoematologia, Fondazione IRCCS Ca' Granda-Ospedale Maggiore Policlinico, Milan, Italy; ${ }^{3}$ Dipartimento di Medicina del Lavoro, Sez. Biometria, Università degli Studi di Milano, Milan, Italy; ${ }^{4}$ U.O. di Ematologia-Centro Trapianti di Midollo, Università degli Studi di Milano, Fondazione IRCCS Ca' Granda-Ospedale Maggiore Policlinico, Milan, Italy and ${ }^{5}$ U.O. di Anatomia Patologica, A.O. San Paolo, Milan, Italy

We investigated the relationship between the International Prognostic Scoring System of the International Working Group for Myelofibrosis Research and Treatment and the European Consensus on grading of bone marrow fibrosis (MF) in patients with primary myelofibrosis. We compared them in 196 consecutive primary myelofibrosis patients (median follow-up 45.7 months; range 7.4-159). International Prognostic Scoring System classified 42 cases as low risk, 73 as intermediate risk-1, 69 as intermediate risk-2, and 12 as high risk; European Consensus on grading of bone marrow fibrosis classified 83 cases as MF-0, 58 as MF-1, 41 as MF-2, and 14 as MF-3. By the time of the analysis, 30 patients $(15.3 \%)$ had died. Overall median survival was 3.8 years (95\% confidence interval: 3.3-4.3). Multivariate analysis confirmed that both scoring systems independently predicted survival, with hazard ratios similar to those provided by univariate analysis (respectively, $2.40(95 \%$ confidence interval: 1.47-3.91) and 2.58 (95\% confidence interval: 1.72-3.89) but the likelihood ratio increased from 19.6 of the International Prognostic Scoring System or 29.0 of the European Consensus on grading of bone MF to 42.3 when both measures were considered together. Analysis of the overall survival curves documented that patients classified as having the most favourable rate with both prognostic scores (ie low risk and MF-0) survive longer than those with only one favourable score (ie low risk but MF $>0$ or MF-0, but International Prognostic Scoring System > low risk). In contrast, those patients classified as having the most unfavourable rate for both scores (high risk and MF-3) have a shorter survival than those with only one unfavourable score (ie high risk but MF $<3$ or MF-3, but International Prognostic Scoring System $<$ high risk). In conclusion, our analysis suggests that better prognostication can be achieved in primary myelofibrosis patients when both systems are used together.

Modern Pathology (2012) 25, 1193-1202; doi:10.1038/modpathol.2012.87; published online 25 May 2012

Keywords: European Consensus on grading of bone marrow fibrosis; International Prognostic Scoring System; primary myelofibrosis

Correspondence: Dr U Gianelli, Pathology Unit, Department of Medicine, Surgery and Dentistry, University of Milan School of Medicine, IRCCS Ca' Granda-Fondazione Ospedale Maggiore Policlinico, Via Francesco Sforza, 35, 20122 Milano, Italy.

E-mail: umberto.gianelli@unimi.it

${ }^{6}$ These authors contributed equally to this work.

Received 3 October 2011; revised 7 December 2011; accepted 9

December 2011; published online 25 May 2012
Several pathological conditions are associated with increased bone marrow fibrosis (MF). In particular, reticulin fibrosis may be evident in many benign situations, including autoimmune and granulomatous diseases, and different tumours, such as lymphoid neoplasms, myelodysplastic syndromes and acute myeloid leukaemia. In contrast, collagen 
fibrosis appears to be more characteristic of the advanced phases of myeloproliferative neoplasms or associated with tumour metastasis to the bone marrow. ${ }^{1,2}$

Assessment of marrow fibrosis has been shown to have clinical and prognostic implication in different neoplasms. In chronic myelogenous leukaemia, this parameter has been demonstrated to have predictive value on therapy and outcome. ${ }^{3}$ In multiple myeloma, increase of bone marrow fibrosis correlates with poorly differentiated plasma cell morphology and mitotic activity and is associated with reduced median survival time. ${ }^{4}$ The grading of bone marrow fibrosis has been reported to have a prognostic significance in primary myelofibrosis,,${ }^{5,6}$ and in a recent study, we have confirmed these data. ${ }^{7}$ Finally, a distinct subgroup of patients with myelodysplastic syndrome has bone marrow fibrosis and this finding is associated with high transfusion requirements and poor prognosis. ${ }^{8}$

During the past two decades, different grading systems have been created to assess bone marrow fibrosis in pathological conditions, most of them deriving from the Bauermeister scale. ${ }^{9} \mathrm{~A}$ few years ago, a panel of expert pathologists published the European Consensus on grading of bone marrow fibrosis. ${ }^{2}$ This scheme consists of a qualitative (reticulin or collagen) and quantitative evaluation of bone marrow fibrosis and distinguishes four increasing categories, ranging from MF-0, which corresponds to normal bone marrow, to MF-3, in which coarse bundles of collagen fibrosis are identifiable with significant osteosclerosis. ${ }^{2}$ This scheme has been added to the updated WHO classification system of tumours of the haematopoietic and lymphoid tissues for the purpose of grading of primary myelofibrosis. ${ }^{10}$

A number of prognostic scoring systems based on clinical variables have been proposed to select at diagnosis high-risk primary myelofibrosis patients who may be eligible for intensive treatments. ${ }^{11-14}$ Three of these (the Mayo, ${ }^{12}$ Cervantes, ${ }^{13}$ and Dupriez $^{14}$ ) have been compared in the same series of patients, and were all found to have significant prognostic value, although the results of the Mayo prognostic scoring system were the most substantiated. ${ }^{12}$ The International Working Group for Myelofibrosis Research and Treatment has recently proposed an International Prognostic Scoring System for primary myelofibrosis, based on five clinical parameters predicting shortened survival: $:^{15}$ age $>65$ years, presence of constitutional symptoms, haemoglobin levels $<10 \mathrm{~g} / \mathrm{dl}$, leukocytes count $>25 \times 10^{9} / \mathrm{l}$, and circulating blast cells $\geq 1 \%$. These variables delineate four risk groups with distinct survival curves: low risk, intermediate risk-1, intermediate risk-2, and high risk.

At present, all primary myelofibrosis prognostic scoring system used by clinicians are based exclusively on clinical parameters and do not consider those pathological changes underlying the clinical factors. However, several papers have clearly demonstrated that pathological alterations of the bone marrow are associated with relevant haematological findings, and that when pre-fibrotic primary myelofibrosis progresses to a more advanced disease, the clinical parameters change correspondingly. ${ }^{5,6}$

Assuming that marrow stromal changes, such as reticulin or collagen fibrosis, and myeloid proliferation represent the pathological alterations of the primary myelofibrosis, grading of bone MF may be considered as a method for directly assessing the disease. In contrast, clinical parameters result from underlying pathological changes, but can also be influenced by extrinsic factors and therefore may represent an indirect method to evaluate the disease.

On the basis of this hypothesis, we investigated in this study the relationship between the International Prognostic Scoring System and European Consensus on grading of bone MF by comparing the two scores in a large series of primary myelofibrosis patients. Our aim was to verify whether greater prognostic knowledge could be acquired using clinical and pathological systems simultaneously.

\section{Patients and methods}

\section{Patients}

The study involved 196 consecutive primary myelofibrosis patients (104 males and 92 females) diagnosed between 1996 and 2008 (median followup 45.7 months, range 7.4-159) at Fondazione IRCCS Ca' Granda-Ospedale Maggiore Policlinico of Milan. All of the cases were reviewed and classified on the basis of the updated WHO classification: of the total cohort, 141 were consistent with early/pre-fibrotic primary myelofibrosis (MF-0 and MF-1) and 55 with the fibrotic stage (MF-2 and MF-3) ${ }^{11}$ Cases of post-polycythaemic and post-essential thrombocythaemia myelofibrosis have been excluded from the study on the basis of the clinical history, the laboratory data, and previous bone marrow biopsies.

Using the European Consensus on grading of bone marrow fibrosis, 83 cases were classified as MF-0, 58 as MF-1, 41 as MF-2, and 14 as MF-3. According to the International Prognostic Scoring System criteria, 42 cases were stratified as low risk, 73 as intermediate risk-1, 69 as intermediate risk-2, and 12 as high risk. Clinical data were available for each patient and all were Philadelphia chromosome-negative. All of the patients gave their informed consent.

\section{Bone Marrow Histology}

Formalin-fixed, paraffin-embedded bone marrow biopsies obtained at the diagnosis were available for all of the patients. Sections were stained with 
haematoxylin-eosin, Giemsa, and Gomori's silver impregnation, in order to evaluate their morphology and bone marrow fibrosis grade.

Reticulin staining was scored by two experienced pathologists (UG, AM) with no knowledge of the clinical data except for the previous primary myelofibrosis diagnosis, using a double-headed microscope and a scale of $0-3$ according to the European Consensus on grading of bone marrow fibrosis. ${ }^{2}$ Agreement was 93\% (182/196 cases) and discordant cases were re-examined until a consensus was obtained.

\section{Statistical Analysis}

Continuous variables were expressed as median in range, and categorical variables expressed as absolute frequency and percentage.

Proportional hazard Cox regression was used to model the effects of European Consensus on grading of bone marrow fibrosis, International Prognostic Scoring System, and their subsequent interaction upon survival. The absence of severe violations of the proportional hazard assumption was ascertained by inspecting a plot of log (cumulative hazard) vs log (survival duration). The two scores were introduced into the model as continuous variables as opposed to categorical variables because the increase in hazard ratio from one category of European Consensus on grading of bone marrow fibrosis or International Prognostic Scoring System to the next was nearly constant. The hazard ratios were calculated using either European Consensus on grading of bone marrow fibrosis MF-0 or International Prognostic Scoring System low risk as a reference in univariate analyses, while both European Consensus on grading of bone marrow fibrosis MF-0 and International Prognostic Scoring System low risk were referenced in multivariate analyses.

Statistical significance was set at a level of $P<0.05$. Data processing and all of the statistical analyses were performed using the SAS software (version 9.1; SAS Institute, Cary, NC, USA).

\section{Results}

\section{Patients}

The study involved a consecutive series of 196 patients with WHO-defined primary myelofibrosis. Table 1 shows their characteristics at the time of diagnosis. Median age was 67 years (range 27-85); 89 patients (45.4\%) were younger than 65 years. The presence of constitutional symptoms was observed in 10 patients $(5.1 \%)$, haemoglobin $\leq 10 \mathrm{~g} / \mathrm{dl}$ in 34 (17.4\%), leukocytes count $>25 \times 10^{9} / 1$ in $7(3.6 \%)$, and $\geq 1 \%$ circulating blast cells in $47(25.8 \%)$.

JAK2 mutational status was known in all of the patients and was positive in 99 cases (59.6\%).
Table 1 Baseline characteristics of the 196 enrolled patients

\begin{tabular}{|c|c|c|}
\hline \multicolumn{3}{|l|}{ Variables } \\
\hline $\begin{array}{l}\text { Age at diagnosis (years)-median } \\
\text { (range) }\end{array}$ & 67 & $(27-85)$ \\
\hline \multicolumn{3}{|l|}{ Age groups-number of patients (\%) } \\
\hline$\leq 65$ years & 89 & $(45.4)$ \\
\hline$>65$ years & 107 & (54.6) \\
\hline Males-number of patients (\%) & 104 & (53.1) \\
\hline $\begin{array}{l}\text { Constitutional symptoms-number of } \\
\text { patients }(\%)\end{array}$ & 10 & $(5.1)$ \\
\hline $\begin{array}{l}\text { Haemoglobin level (g/dl) —median } \\
\text { (range) }\end{array}$ & 12.8 & $(6.2-17.1)$ \\
\hline $\begin{array}{l}\text { Haemoglobin level }(\leq 10 \mathrm{~g} / \mathrm{dl})- \\
\text { number of patients }(\%)\end{array}$ & 34 & $(17.4)$ \\
\hline $\begin{array}{l}\text { White blood cells count }\left(\times 10^{9} / 1\right)- \\
\text { median (range) }\end{array}$ & 8.8 & $(0.42-41.6)$ \\
\hline $\begin{array}{l}\text { White blood cells count }\left(>25 \times 10^{9} / 1\right) \\
\text { - number of patients }(\%)\end{array}$ & 7 & $(3.6)$ \\
\hline $\begin{array}{l}\text { Platelets count }\left(\times 10^{9} / 1\right)-\text { median } \\
\text { (range) }\end{array}$ & 639.5 & $(4-1742)$ \\
\hline $\begin{array}{l}\text { Peripheral blast cells } \geq 1 \% \text {-number of } \\
\text { patients }(\%)\end{array}$ & 47 & $(25.8)$ \\
\hline $\begin{array}{l}\text { Lactate dehydrogenase-median } \\
\text { (range) (U/l) }\end{array}$ & 594 & $(281-3897)$ \\
\hline Splenomegaly (cm)-median (range) & 2 & $(0-22)$ \\
\hline Hepatomegaly $(\mathrm{cm})$-median (range) & 2 & $(0-9)$ \\
\hline \multicolumn{3}{|l|}{ JAK2 } \\
\hline Negative (\%) & 67 & $(40.4)$ \\
\hline Positive $(\%)$ & 99 & $(59.6)$ \\
\hline Deaths_number of patients (\%) & 30 & (15.3) \\
\hline
\end{tabular}

Table 2 Stratification of 196 patients with primary myelofibrosis according to the European Consensus on grading of bone marrow fibrosis (MF) and the risk groups of the International Prognostic Scoring System (low = low risk; int-1=intermediate-1 risk; int$2=$ intermediate-2 risk; high $=$ high risk)

\begin{tabular}{|c|c|c|c|c|c|}
\hline \multirow[t]{2}{*}{ Bone $M F$} & \multicolumn{4}{|c|}{ International Prognostic Scoring System } & \multirow[t]{2}{*}{ Total } \\
\hline & Low & Int-1 & Int-2 & High & \\
\hline MF-0 & 27 & 36 & 17 & 3 & $83(42.3 \%)$ \\
\hline MF-1 & 11 & 21 & 24 & 2 & $58(29.6 \%)$ \\
\hline MF-2 & 3 & 12 & 22 & 4 & $41(20.9 \%)$ \\
\hline MF-3 & 1 & 4 & 6 & 3 & $14(7.2 \%)$ \\
\hline Total & $42(21.4 \%$ & $7337.3 \%$ & $69(35.2 \%)$ & $12(6.1 \%)$ & 196 \\
\hline
\end{tabular}

Patient Classification with the International
Prognostic Scoring System and the European
Consensus on Grading of Bone Marrow Fibrosis

All of the patients were classified according to the International Prognostic Scoring System ${ }^{16}$ and the European Consensus on grading of bone marrow fibrosis $^{2}$ (Table 2). Examples of cases with different degrees of fibrosis are reported in Figure 1. Interestingly, only 73 out of 196 patients (37\%) were classified as having a corresponding score by both systems. Moreover, 4 out of $42(9.5 \%)$ patients were at low risk according to International Prognostic Scoring System, but MF-2 or MF-3 according to the European Consensus on grading of bone marrow 
Hematoxylin-Eosin
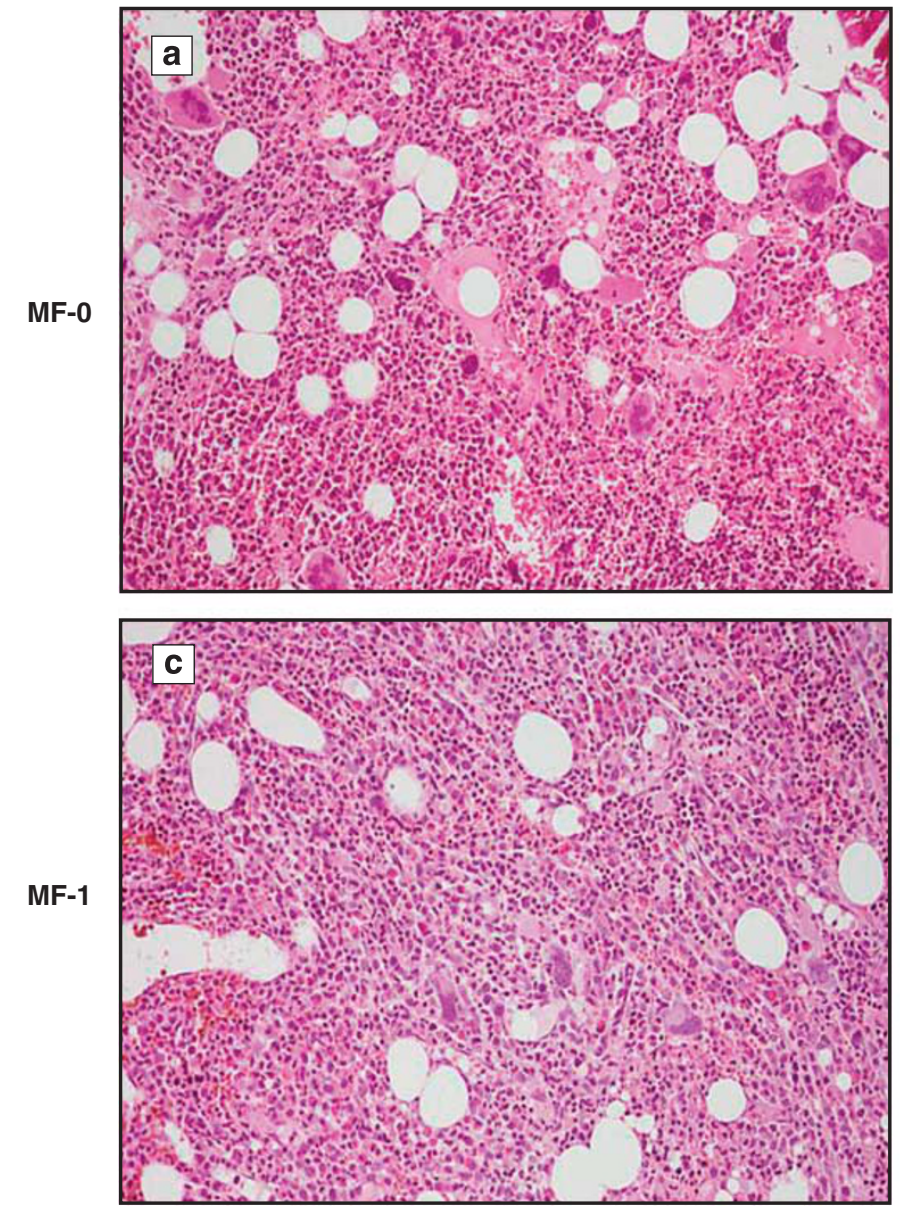

Gomori
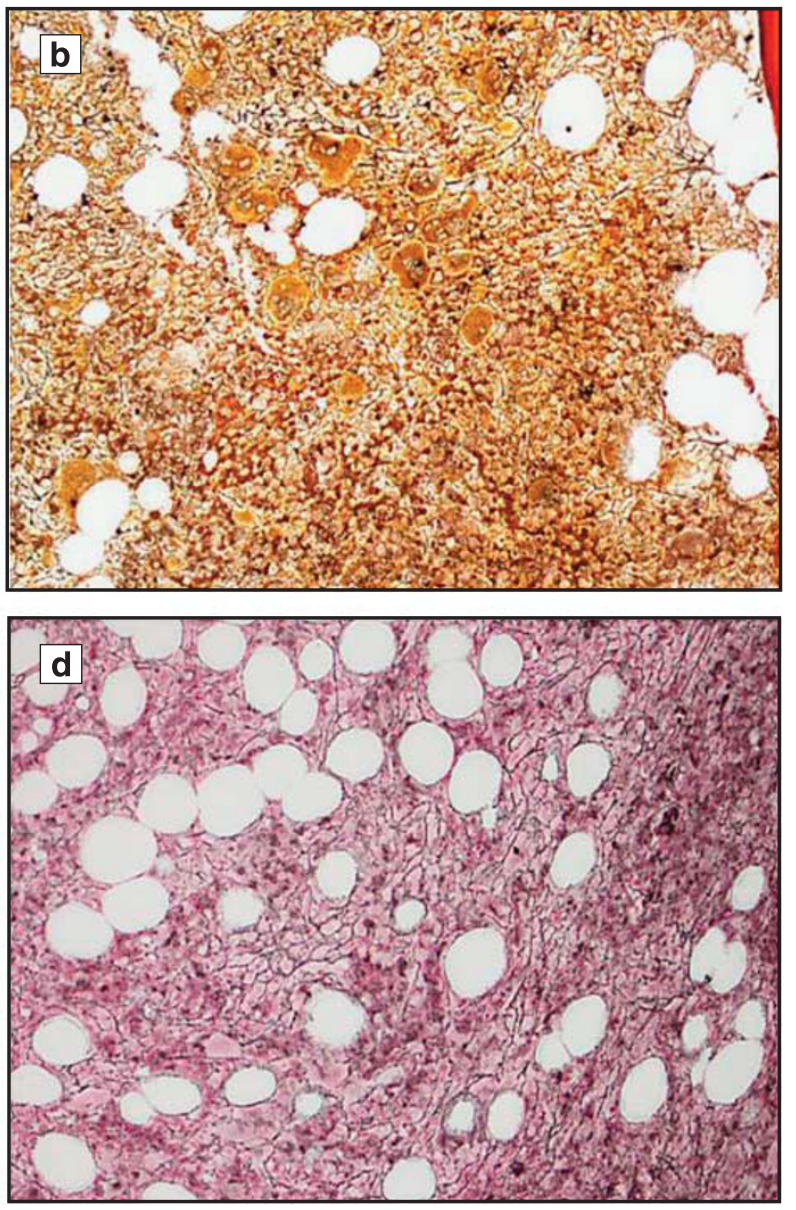

Figure 1 Examples of morphological features of primary myelofibrosis and the grading of bone marrow fibrosis (haematoxylin-eosin and Gomori, $\times 20$ ). (a, b) Pre-fibrotic primary myelofibrosis (MF-0): scattered linear reticulin fibres with no intersections. (c, d) Early-stage primary myelofibrosis (MF-1): loose network of reticulin fibres with many intersections, especially in perivascular areas. (e, f) Fibrotic stage primary myelofibrosis (MF-2): diffuse and dense increase in reticulin fibres, with extensive intersections and occasionally with focal bundles of collagen. (g, h) Fibrotic stage primary myelofibrosis (MF-3): diffuse and dense increase in reticulin fibres, with extensive intersections and coarse bundles of collagen.

fibrosis, and 12 patients classified as being at high risk by International Prognostic Scoring System were equally distributed among the four grades of European Consensus on grading of bone marrow fibrosis.

When International Prognostic Scoring System and European Consensus on grading of bone marrow fibrosis grades increased (respectively, from low to high risk and from MF-0 to MF-3), the degree of splenomegaly, hepatomegaly, lactate dehydrogenase values, the presence and percentage of peripheral blasts, the occurrence of acute myeloid leukaemia, and the need for blood transfusions increased, whereas platelets count decreased. Bearing in mind that the number of patients with the highest scores of both prognostic systems was limited (International Prognostic Scoring System high risk, $n=12$ and European Consensus on grading of bone marrow fibrosis MF-3, $n=14$ ), the changes (increased or decreased) in the values of clinical variables observed in International Prognostic Scoring System groups appeared to be quite similar to those in the European Consensus on grading of bone marrow fibrosis groups (Figure 2).

However, we observed a high degree of variability of the levels of two clinical parameters pertaining to disease severity, namely lactate dehydrogenase and platelets count, in each International Prognostic Scoring System group, when varying the grade of bone marrow fibrosis, suggesting a clinical heterogeneity within International Prognostic Scoring System prognostic groups. For example, lactate dehydrogenase levels in 73 patients with International Prognostic Scoring System low risk ranged from 470 to $1200 \mathrm{U} / \mathrm{l}$ when European Consensus on grading of bone marrow fibrosis changed from MF-0 to MF-3. Similarly, the platelet counts in 69 patients with International Prognostic Scoring System intermediate risk-1 ranged from 179 to $736 \times 10^{9} / \mathrm{l}$ when European Consensus on grading of bone marrow fibrosis changed from MF-0 to MF-3 (Figure 3). 

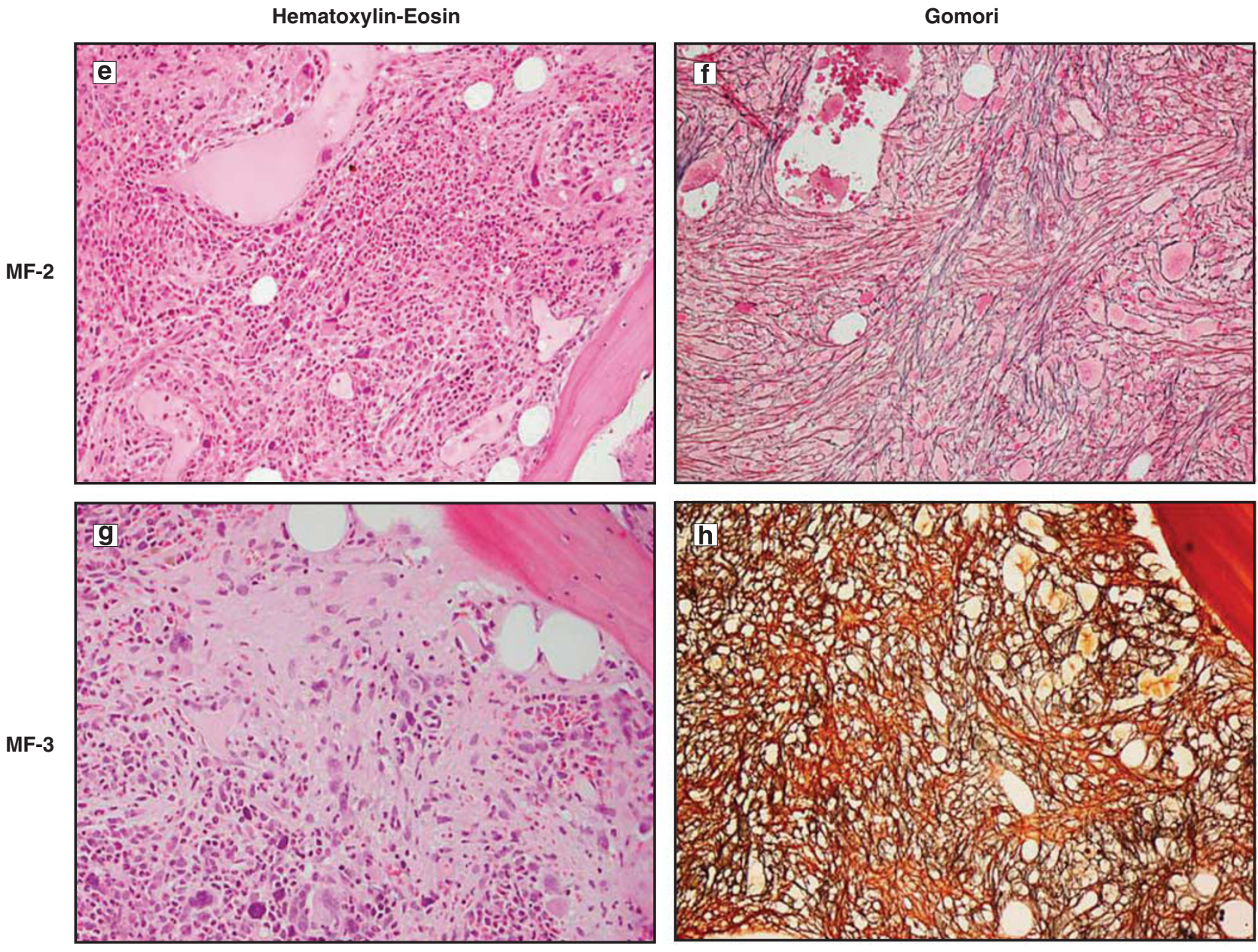

Figure 1 Continued.

\section{Patients Stratification Using Both International Prognostic Scoring System and European Consensus on Grading of Bone of Marrow Fibrosis is a Stronger Predictor of Survival}

By the time of the analysis, 30 patients (15.3\%) had died. Overall median survival was 3.8 years $(95 \%$ confidence interval: 3.3-4.3). Univariate analysis confirmed the adverse prognostic relevance of International Prognostic Scoring System and European Consensus on grading of bone marrow fibrosis; as expected, higher International Prognostic Scoring System scores and higher European Consensus on grading of bone marrow fibrosis grades predicted poor survival. The hazard ratio of mortality increased by 2.7 times (95\% confidence interval: $1.7-4.4)$ and by 2.7 (95\% confidence interval: 1.9-3.9), respectively, for a unit increase in the score (Table 3a). The survival curves of the four International Prognostic Scoring System groups were similar to those of the four European Consensus on grading of bone marrow fibrosis groups (Figure $4 \mathrm{a}$ and $\mathrm{b}$ ).

To verify whether European Consensus on grading of bone marrow fibrosis helped to differentiate the prognosis of the patients with the same International Prognostic Scoring System score (and vice versa), a proportional hazard Cox regression model was fitted that included International Prognostic Scoring System, European Consensus on grading of bone MF, and their interaction. The interaction term was removed because it was not statistically significant (hazard ratio $1.28 ; P=0.35$ ). The model including only International Prognostic Scoring System or European Consensus on grading of bone marrow fibrosis as an explanatory variable confirmed that both independently predict survival. In fact, the likelihood ratio of the multivariate model (42.3) is nearly equivalent to the sum of the likelihood ratio of the two univariate models (19.6 and 29.0), and hazard ratios values of the multivariate model (hazard ratio $=2.4$ when International Prognostic Scoring System is int-1 (95\% confidence interval: 1.5-3.9) and hazard ratio $=2.6$ when European Consensus on grading of bone marrow fibrosis is MF-1 (95\% confidence interval: 1.7-3.9)) are close to that of univariate model (Table $3 \mathrm{~b}$ ).

The simultaneous use of both scoring systems allowed for a more precise prediction of survival. In fact, Table $3 \mathrm{~b}$ shows that for a given value of one 

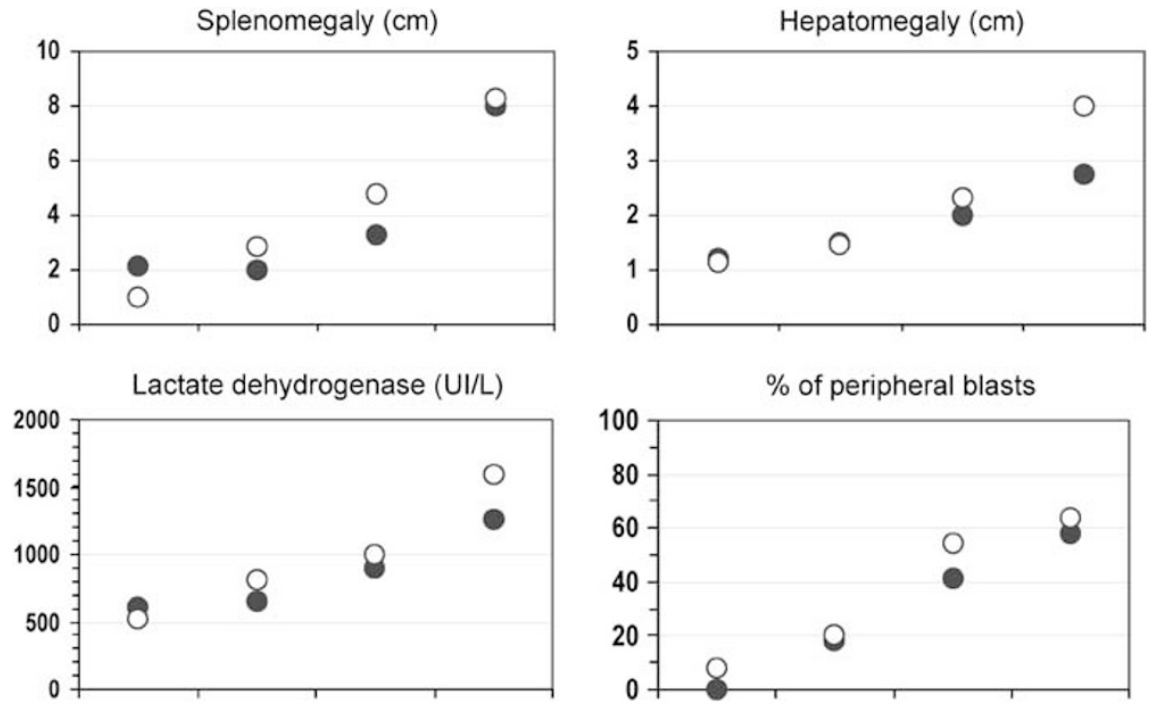

$\%$ of patients with peripheral blasts $\geq 1 \%$
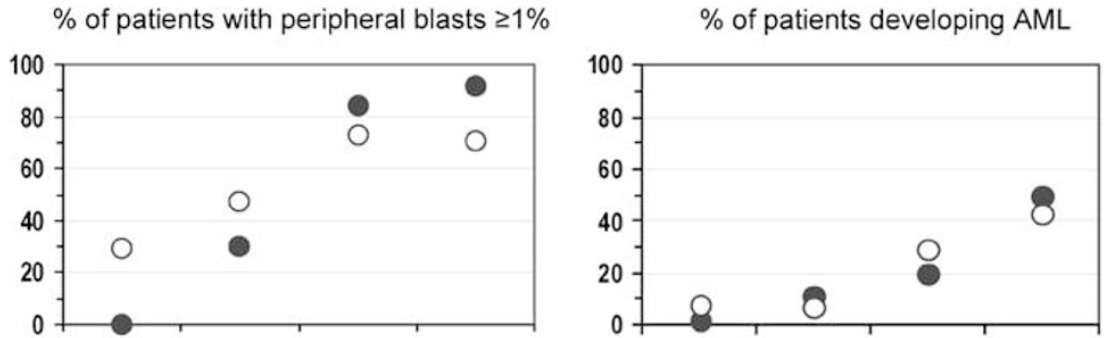

$\%$ of patients needing blood transfusion

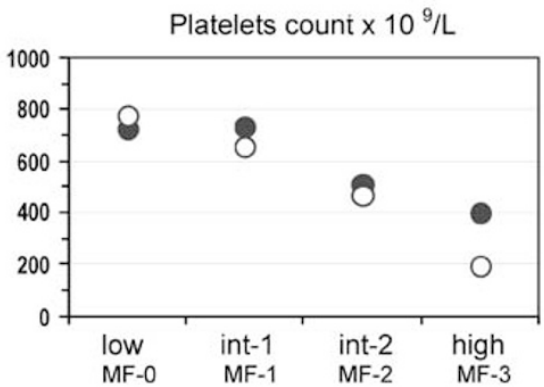

Figure 2 Changes of clinical parameters in patients with primary myelofibrosis with different scores of the International Prognostic Scoring System (low=low risk; int-1=intermediate-1 risk; int-2=intermediate-2 risk; high=high risk) (๑) and the European Consensus on grading of bone marrow fibrosis $(\mathrm{MF})(\bigcirc)$.

scoring system, the hazard ratio increases with increasing values of the other scoring system; for example, the hazard ratio when International Prognostic Scoring System is the only explanatory variable (International Prognostic Scoring System int-1) is 2.74 (Table 3a), but when International Prognostic Scoring System is intermediate risk-1 and European Consensus on grading of bone marrow fibrosis is MF-2 hazard ratio increases to 16.0 (95\% confidence interval: $6.2-41.3)$ and to 41.4 (95\% confidence interval: 11.1-154.0) if European Consensus on grading of bone marrow fibrosis is MF-3 (Table 3b).

This implies that patients classified as being at low risk by both prognostic scores (International Prognostic Scoring System low risk and European Consensus on grading of bone marrow fibrosis MF-0) survive longer than those classified as low risk on the basis of only one score (ie International Prognostic Scoring System low risk but European Consensus on grading of bone marrow fibrosis $\mathrm{MF}>0$ or European Consensus on grading of bone marrow fibrosis MF-0, but International Prognostic Scoring System > low risk). In contrast, those patients classified as being at high risk using both scores (International Prognostic Scoring System high risk and European Consensus on grading of bone marrow fibrosis MF-3) have a shorter survival than those with only one unfavourable score (ie International Prognostic Scoring System high risk but European Consensus on grading of bone marrow fibrosis MF $<3$ or European Consensus on grading of bone marrow fibrosis MF-3, but International Prognostic Scoring System < high risk) (Figure 4c-f). 


\section{Discussion}

In this study, we compared two scoring systems, one clinical (International Prognostic Scoring System) and one pathological (European Consensus on grading of bone marrow fibrosis), in a series of 196 primary myelofibrosis, to verify whether the prognostic capacity in terms of survival can be improved by concurrent use of both systems.

Univariate analysis confirmed that higher International Prognostic Scoring System scores and higher bone marrow fibrosis grades independently predicted poor survival: the overall survival in patients with higher International Prognostic Scoring System or bone marrow fibrosis scores was
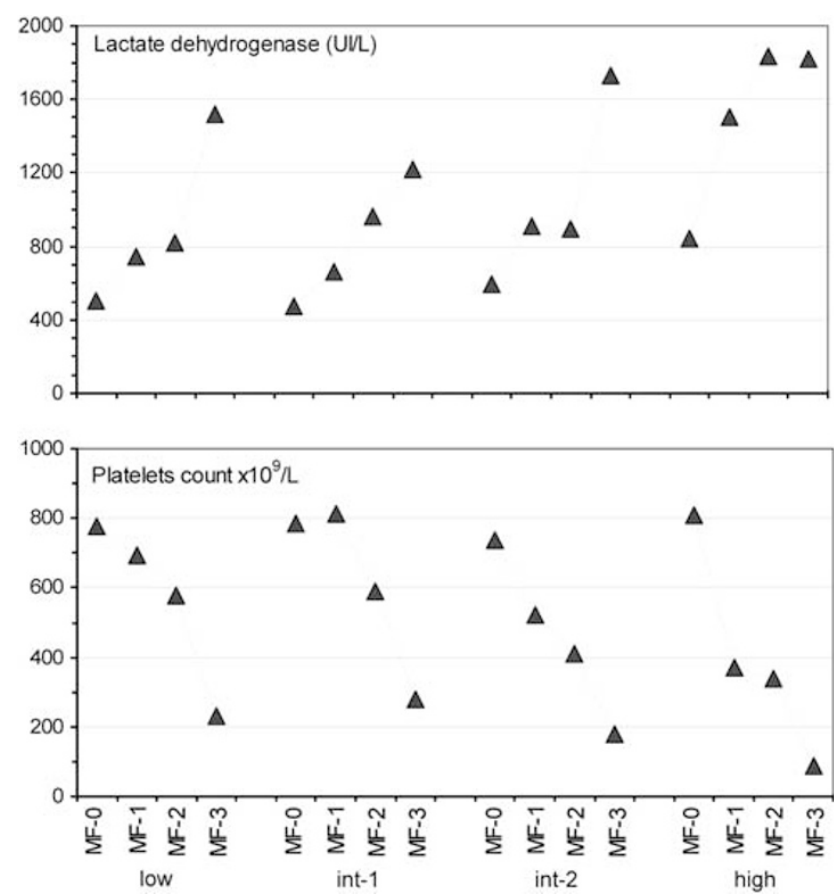

Figure 3 Lactate dehydrogenase levels (top) and platelet counts (bottom) in patients with the same International Prognostic Scoring System scores (low $=$ low risk; int-1 = intermediate-1 risk; int-2 = intermediate- 2 risk; high $=$ high risk) but different European Consensus on grading of bone marrow fibrosis grades (MF). significantly worse than in those with lower scores. Moreover, our data demonstrated that the simultaneous use of European Consensus on grading of bone marrow fibrosis and International Prognostic Scoring System prognostic systems allows a better prediction of survival. These results may have considerable practical implications in defining patient prognosis at the time of the diagnosis.

For example, the hazard ratio $=1$ of patients with an International Prognostic Scoring System low risk becomes 17.2 if associated with European Consensus on grading of bone marrow fibrosis MF-3, while the hazard ratio $=7.5$ of patients with an International Prognostic Scoring System intermediate risk-2 becomes 99.3 when bone marrow fibrosis corresponds to European Consensus on grading of bone marrow fibrosis MF-3 (Table 3b). This concept is clearly comprehensible when focusing on the overall survival curves (Figure 3). In fact, while considering separately the two prognostic scoring systems, the survival curves of the four International Prognostic Scoring System groups were similar to those of the four European Consensus on grading of bone marrow fibrosis groups (Figure 4a and b). On the other hand, the simultaneous use of both scoring systems enables the identification of patients at extremely low risk (International Prognostic Scoring System low risk and European Consensus on grading of bone marrow fibrosis MF-0), who survive longer, and those at extremely high risk (International Prognostic Scoring System high risk and European Consensus on grading of bone marrow fibrosis MF-3), who have a shorter survival (Figure 4c and f).

Primary myelofibrosis is a clinically heterogeneous disease with differences in presentation and evolution. The median overall survival of patients varies widely; some of them die shortly after diagnosis, while others survive for 20 years or more. ${ }^{16}$

In this context, one should be aware that little more than $70 \%$ of our cohort of patients was consistent with early stages of primary myelofibrosis in displaying little to no signs of marrow fibrosis, and therefore showed a more favourable progno-

Table 3a Univariate analysis: hazard ratios (95\% confidence interval) and likelihood ratios (LRs) for International Prognostic Scoring System (low = low risk; int-1 = intermediate-1 risk; int-2 = intermediate-2 risk; high = high risk) and for European Consensus on grading of bone marrow fibrosis (MF)

Univariate analysis

International Prognostic Scoring System

\begin{tabular}{|c|c|c|c|c|}
\hline Low & Int-1 & Int-2 & High & $L R$ \\
\hline 1.00 & $2.74(1.72-4.38)$ & $7.52(4.71-12.00)$ & $20.61(12.90-32.93)$ & 19.6 \\
\hline & & Bone MF & & \\
\hline MF-0 & MF-1 & $\mathrm{MF}-2$ & MF-3 & LR \\
\hline 1.00 & $2.70(1.85-3.93)$ & $7.27(4.98-10.61)$ & $19.61(13.44-28.60)$ & 29.0 \\
\hline
\end{tabular}



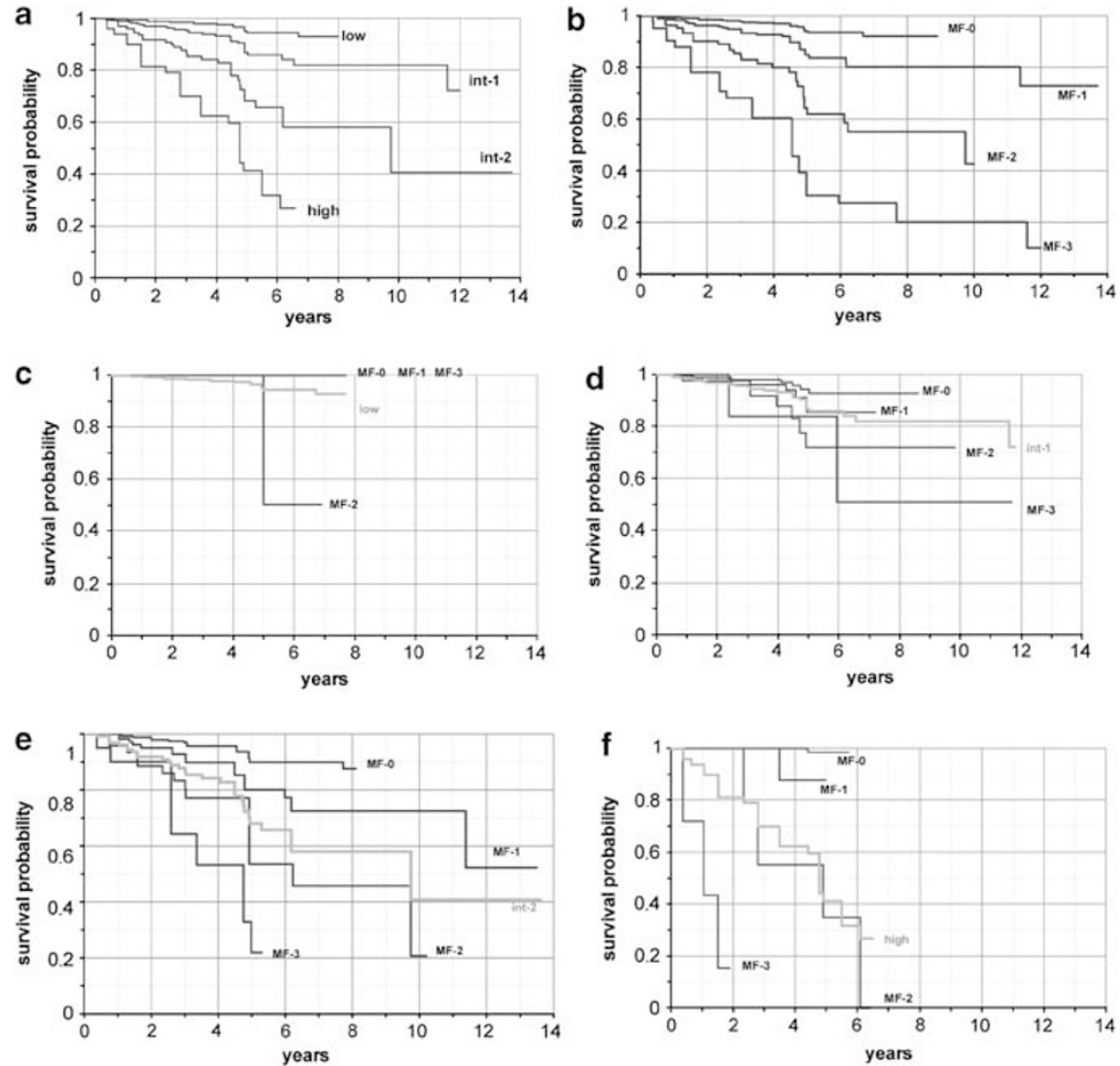

Figure 4 Survival probabilities related to different International Prognostic Scoring System scores (low =low risk; int-1=intermediate- 1 risk; int-2 = intermediate-2 risk; high = high risk) (a), different European Consensus on grading of bone marrow fibrosis grades (MF) (b) and when International Prognostic Scoring System and European Consensus on grading of bone marrow fibrosis are applied simultaneously (c-f).

Table 3b Multivariate analysis: hazard ratios (95\% confidence interval) for combined International Prognostic Scoring System (low = low risk; int-1 = intermediate- 1 risk; int-2 = intermediate- 2 risk; high $=$ high risk) and European Consensus on grading of bone marrow fibrosis (MF) scores

Multivariate analysis $(L R=42.3)$

Bone MF

International Prognostic Scoring System

\begin{tabular}{ccccc} 
& Low & Int-1 & Int-2 & High \\
\cline { 2 - 5 } & & & & \\
MF-0 & 1.00 & $2.4(1.5-3.9)$ & $5.8(2.2-15.3)$ & $13.8(3.2-59.8)$ \\
MF-1 & $2.6(1.7-3.9)$ & $6.2(3.3-11.7)$ & $14.9(5.2-42.7)$ & $35.7(7.8-162.6)$ \\
MF-2 & $6.7(2.9-15.1)$ & $16.0(6.2-41.3)$ & $38.4(10.8-136.2)$ & $92.3(17.4-489.2)$ \\
MF-3 & $17.2(5.0-58.8)$ & $41.4(11.1-154.0)$ & $99.3(20.9-471.5)$ & $238.4(35.7-1590.5)$ \\
\hline
\end{tabular}

sis. $^{5-7}$ This over-representation is explainable with the practice, in ours institutions, to perform bone marrow biopsies during initial patient evaluation, allowing a better disease classification and a early stage identification.

Difficulties in prognosis prediction have stimulated interest in the development of many prognostic scoring systems. ${ }^{11-17}$ At present, primary myelofibrosis treatment is essentially palliative; allogenic stem cell transplantation is increasingly being used, and newer drugs (such as anti-JAK2targeted drugs) are being tested in such patients. ${ }^{17-19}$ Although mortality is less frequent among the patients undergoing reduced-intensity conditioning allogenic stem cell transplantation than in those undergoing conventional allogenic stem cell transplantation, there is still some associated mortality and morbidity and the prognostic stratification of primary myelofibrosis patients is thus important when making treatment decisions. 
In a previous study, we tested the prognostic significance of the European Consensus on grading of bone marrow fibrosis system in primary myelofibrosis patients, and compared it with all of the validated prognostic scoring systems (Mayo, ${ }^{12}$ Dingli, ${ }^{20}$ Cervantes, ${ }^{13}$ and Dupriez $^{14}$ ). The results showed that the histological model was significantly associated with differences in overall survival and, unlike the others, clearly distinguished the overall survival of intermediate- and high-risk patients. The International Working Group for Myelofibrosis Research and Treatment has recently proposed the International Prognostic Scoring System, which is structurally based on five clinical parameters (all of which are readily available at the time of diagnosis), for predicting shortened survival. ${ }^{15}$ The International Prognostic Scoring System identifies four prognostic groups that are clearly different in terms of survival: low- and high-risk patients (who each made up more than one-fifth of the study population) had a median survival of about 11 and 2 years, respectively, whereas for patients in the two intermediate-risk categories, it was 8 and 4 years. The International Prognostic Scoring System had more discriminatory power than the previous scoring systems as well as high levels of reproducibility and predictive accuracy.

To the best of our knowledge, our study represents the first attempt to compare this clinical prognostic scoring system with a pathological feature characteristic of primary myelofibrosis. Our results, while considering the fact that the number of patients with the highest scores of both prognostic systems is limited, demonstrated that the prognostic stratification that can be obtained by simultaneously using International Prognostic Scoring System and European Consensus on grading of bone marrow fibrosis may more readily indentify patients with a worse prognosis and are thus candidates for more aggressive therapy.

Moreover, an International Working Group for Myelofibrosis Research and Treatment study proposed a Dynamic International Prognostic Scoring System, designed to predict the survival of primary myelofibrosis patients in a time-dependent manner, assuming that it was possible to assess patient prognosis at any time during the clinical course of the disease. ${ }^{21}$ The need to monitor the disease dynamically is related to the possibility of identifying the best time to refer a given patient to the most appropriate therapeutic choice. The fact that primary myelofibrosis is a continuously evolving disorder requires the use of a dynamic prognostic scoring system such as the International Working Group for Myelofibrosis Research and Treatment. On the other hand, the extremely low number of complications related to the bone marrow biopsy procedure, which in the more recent series is lower than $0.1-0.5 \%,{ }^{22}$ supports the execution of subsequent bone marrow biopsies to evaluate directly the evolution of marrow fibrosis. This procedure could allow monitoring the progressive stromal changes that become difficult to reverse in advanced stage (collagen fibrosis) and contribute to identifying the most appropriate therapeutic choice.

In conclusion, our findings suggest that an effective approach in the evaluation of a primary myelofibrosis patient prognosis could be the use of both International Prognostic Scoring System and European Consensus on grading of bone marrow fibrosis not only at diagnosis, but also whenever decisions must be made concerning specific treatment options.

\section{Acknowledgement}

This work was supported in part by grant from the Fondazione Matarelli per lo Studio e la Cura delle Malattie del Sangue and Fondazione Beat-Leukemia Dr Alessandro Cevenini (beat-leukemia.org).

\section{Disclosure/conflict of interest}

The authors declare no conflict of interest.

\section{References}

1 Kuter DJ, Bain B, Mufti G, et al. Bone marrow fibrosis: pathophysiology and clinical significance of increased bone marrow stromal fibres. Br J Haematol 2007;139: 351-362.

2 Thiele J, Kvasnicka HM, Facchetti F, et al. European Consensus on grading of bone marrow fibrosis and assessment of cellularity. Haematologica 2005;90: 1128-1132.

3 Kvasnicka HM, Thiele J, Schmitt-Graeff A, et al. Bone marrow features improve prognostic efficiency in multivariate risk classification of chronic-phase $\mathrm{Ph}$ (1+) chronic myelogenous leukaemia: a multicenter trial. J Clin Oncol 2001;19:2994-3009.

4 Subramanian R, Basu D, Dutta TK. Significance of bone marrow fibrosis in multiple myeloma. Pathology 2007; 39:512-515.

5 Thiele J, Kvasnicka HM. Hematopathologic findings in chronic idiopathic myelofibrosis. Semin Oncol 2005; 32:380-394.

6 Thiele J, Kvasnicka HM. Grade of bone marrow fibrosis is associated with relevant haematological findings-a clinicopathological study on 865 patients with chronic idiopathic myelofibrosis. Ann Hematol 2006;85:226-232.

7 Vener C, Fracchiolla NS, Gianelli U, et al. Prognostic implications of the European Consensus for grading bone marrow fibrosis in chronic idiopathic myelofibrosis. Blood 2008;111:1862-1865.

8 Della Porta MG, Malcovati L, Boveri E, et al. Clinical relevance of bone marrow fibrosis and CD34-positive cell clusters in primary myelodysplastic syndromes. J Clin Oncol 2009;27:754-762.

9 Bauermeister DE. Quantitation of bone marrow reticulin-a normal range. Am J Clin Pathol 1971;56:24-31.

10 Thiele J, Kvasnicka HM, Tefferi A, et al. Primary myelofibrosis. In: Swerdlow SH, Campo E (eds). WHO 
Classification of Tumours of the Haematopoietic and Lynphoid Tissues, 4th edn. International Agency for Research on Cancer: Lyon 2008, pp 44-47.

11 Barosi G. Myelofibrosis with myeloid metaplasia: diagnostic definition and prognostic classification for clinical studies and treatment guidelines. J Clin Oncol 1999;17:2954-2970.

12 Tefferi A, Huang J, Schwager S, et al. Validation and comparison of contemporary prognostic models in primary myelofibrosis: analysis based on 334 patients from a single institution. Cancer 2007;109:2083-2088.

13 Cervantes F, Pereira A, Esteve J, et al. Identification of 'shortlived' and 'long-lived' patients at presentation of idiopathic myelofibrosis. Br J Haematol 1997;97: 635-640.

14 Dupriez B, Morel P, Demory JL, et al. Prognostic factors in agnogenic myeloid metaplasia: a report on 195 cases with a new scoring system. Blood 1996;88:1013-1018.

15 Cervantes F, Dupriez B, Pereira A, et al. New prognostic scoring system for primary myelofibrosis based on a study of the International Working Group for Myelofibrosis Research and Treatment. Blood 2009;113:2895-2901.
16 Tefferi A. Myelofibrosis with myeloid metaplasia. N Engl J Med 2000;342:1255-1265.

17 Barosi G, Viarengo G, Pecci A, et al. Diagnostic and clinical relevance of the number of circulating CD34(+) cells in myelofibrosis with myeloid metaplasia. Blood 2001;98:3249-3255.

18 Tefferi A. New insights into the pathogenesis and drug treatment of myelofibrosis. Curr Opin Hematol 2006; 13:87-92.

19 Hennessy BT, Thomas DA, Giles FJ, et al. New approaches in the treatment of myelofibrosis. Cancer 2005;103:32-43.

20 Dingli D, Schwager SM, Mesa RA, et al. Prognosis in transplant-eligible patients with agnogenic myeloid metaplasia. Cancer 2006;106:623-630.

21 Passamonti F, Cervantes F, Vannucchi AM, et al. A dynamic prognostic model to predict survival in primary myelofibrosis: a study by the IWG-MRT (International Working Group for Myeloproliferative Neoplasms Research and Treatment). Blood 2010;115: 1703-1708.

22 Bain BJ. Bone marrow biopsy morbidity and mortality: 2002 data. Clin Lab Haematol 2004;26:315-318. 